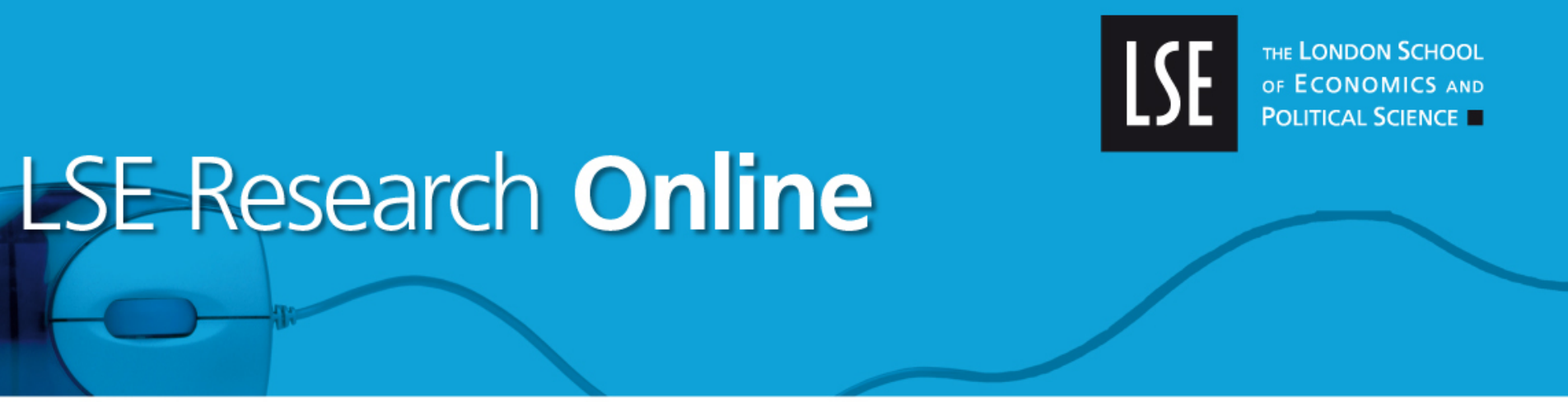

James Nguyen and Roman Frigg

\title{
Mathematics is not the only language in the book of nature
}

\section{Article (Accepted version) (Refereed)}

\author{
Original citation: \\ Nguyen, James and Frigg, Roman (2017). Mathematics is not the only language in the book of \\ nature. Synthese ISSN 0039-7857
}

DOI: $\underline{10.1007 / s 11229-017-1526-5}$

(c) Springer Science+Business Media B.V. 2017

This version available at: http://eprints.Ise.ac.uk/83732/

Available in LSE Research Online: November 2017

LSE has developed LSE Research Online so that users may access research output of the School. Copyright (c) and Moral Rights for the papers on this site are retained by the individual authors and/or other copyright owners. Users may download and/or print one copy of any article(s) in LSE Research Online to facilitate their private study or for non-commercial research. You may not engage in further distribution of the material or use it for any profit-making activities or any commercial gain. You may freely distribute the URL (http://eprints.Ise.ac.uk) of the LSE Research Online website.

This document is the author's final accepted version of the journal article. There may be differences between this version and the published version. You are advised to consult the publisher's version if you wish to cite from it. 


\title{
Mathematics is not the only language in the book of nature
}

\author{
James Nguyen ${ }^{1}$ and Roman Frigg ${ }^{2}$
}

\begin{abstract}
How does mathematics apply to something non-mathematical? We distinguish between a general application problem and a special application problem. A critical examination of the answer that structural mapping accounts offer to the former problem leads us to identify a lacuna in these accounts: they have to presuppose that target systems are structured and yet leave this presupposition unexplained. We propose to fill this gap with an account that attributes structures to targets through structure generating descriptions. These descriptions are physical descriptions and so there is no such thing as a solely mathematical account of a target system.
\end{abstract}

Key words: Application of mathematics; structure; mapping account; representation; isomorphism; physical descriptions.

\section{Introduction}

Much of modern science is highly mathematized. Physicists represent quantum phenomena with Hilbert spaces; population biologists represent predator-prey systems using coupled differential equations; and economists represent the beliefs and desires of human agents with real-valued functions. The question then is: how does mathematics apply to the physical and social worlds? This question has been on the horizon at least since Galileo (1623/1957, p. 238) noted that the book of nature was written in the language of mathematics, and it acquired notoriety when Wigner declared that the "unreasonable effectiveness of mathematics" in science was "something bordering on the mysterious and that there is no explanation for it" (1960, p. 2). The question is central to the philosophy of science but has implications beyond, for example in the philosophy of mathematics where the Quine-Putnam indispensability argument takes the successful application of mathematics in science to deliver ontological conclusions regarding the status of mathematical entities.

A prominent answer to the question has emerged within the structuralist approach to the nature of scientific theories. The basic idea of this account, which Pincock (2004) aptly calls the mapping account, is that a theory provides mathematical structures and that these structures apply to the physical world in virtue of the existence of a structure preserving mapping (henceforth a morphism) that relates the mathematical structure with the structure of the

\footnotetext{
1 jnguyen4@nd.edu, History and Philosophy of Science Program, University of Notre Dame, Notre Dame, Indiana, USA and Centre for Philosophy of Natural and Social Science, London School of Economics and Political Science, London, UK.

${ }^{2}$ r.p.frigg@lse.ac.uk, Department of Philosophy, Logic and Scientific Method, London School of Economics and Political Science, London, UK and Centre for Philosophy of Natural and Social Science, London School of Economics and Political Science, London, UK.
} 
physical system(s) to which the theory applies. The aim of this paper is to examine this account of the application of mathematics. Our conclusion will be that it offers only a partial answer. For such morphisms to be well-defined, physical systems need to exhibit structures and without an account of where these structures come from the account remains incomplete. In this paper we provide a positive answer, namely that these structures are the result of abstracting away the physical aspects of what we call structure generating descriptions: descriptions of physical systems that refer to physical objects and physical properties and relations. We call this the extensional abstraction account.

We proceed as follows. In Section 2 we point out that there is no such thing as "the" problem of the application of mathematics and distinguish between multiple intertwined problems concerning the use of mathematics in science. In Section 3 we introduce the mapping account and present what we take to be the strongest formulation of it. In Section 4 we examine existing suggestions of where the physical structures needed for the mapping account might be found, and argue that they don't stand up to scrutiny. In Section 5 we present a suggestion of how to fill this gap, and in Section 6 we offer concluding remarks concerning mathematical explanation, nominalism, and how our account could be put to use in the context of philosophies of mathematics other than structuralism.

\section{Defining the Problem}

Representations of target systems offered by scientific models and theories crucially involve mathematical structures. ${ }^{3}$ These mathematical structures are applied to physical systems in a way that allows scientists to convert reasoning about the structures into reasoning about the systems to which they apply. Consider the simple example of a population of rabbits. Suppose you want to know how fast the population grows and to this end you construct a mathematical model of the population. One way to reason about such a population is to count the rabbits at a particular time, observe their breeding behaviour, and then formulate a rule about how the population grows. A simple rule is that the rabbits form discrete generations and that the population doubles every time a new generation appears (for instance every six months). Under these assumptions the growth of the population is given by the rule $n_{t+1}=2 n_{t}$ where $n_{t}$ is the size of the population at time $t$. It follows from this rule that if one has two rabbits now, one will have 32 rabbits in two years time. ${ }^{4}$

\footnotetext{
${ }^{3}$ For the purpose of this paper it is not necessary to take a stance on whether models or theories are the units of scientific representation, nor does it matter whether models should be identified with mathematical structures (for a discussion of these issues see our (2017)). Our point of departure here is the hopefully uncontroversial observation that whatever does the representing often involves mathematics.

${ }^{4}$ We note that our choice of example is inline with much of the recent discussion on the applicability of mathematics where examples from biology have occupied centre stage; $c f$. Baker's cicadas (2005), van Fraassen's deer counting (2008, Chapter 11), and Lyon and Colyvan's bees (2008).
} 
In formulating this model, a mathematical structure, in this case the natural numbers with a particular function defined on them, has been applied to a physical system, and this made it possible to convert reasoning about the structure into reasoning about the physical system itself. This invites the question of what is involved in doing this. Steiner (1998, pp. 24-47) points out that the question about the application of mathematics actually breaks down into two questions that need to be disentangled. We agree with this observation and therefore distinguish between what we call the general application problem and the special application problem.

The general application problem is this: in virtue of what does a mathematical structure apply to a target system? Two qualifications are needed. First, the structure referred to in the question could be any mathematical structure. The emphasis of question is: how does mathematics hook onto something that (at least on the face of) it isn't mathematical, namely a part of the physical world. What general conditions have to fall into place for this to happen? Second, there is an ambiguity in whether "apply" is a success term or not. There are numerous examples where mathematics can be applied to a physical system, but in such a way that the claims that the mathematics can be used to generate about the physical system are false. ${ }^{5}$ The above example is a case in point as the equation implies that the population continues to grow indefinitely, but of course the population won't grow indefinitely because limited resources will put a bound on its size. 6 To regiment usage, we will use "apply" (and its cognates) in a way that is neutral with respect to whether or not the application at hand generates true or false claims. Where the application generates truths we use the phrase "successful application" and where it generates falsehoods the phrase "unsuccessful application". But both types of cases are still instances of mathematics being applied to the physical world.

As our first qualification suggests, there is another question one can ask, namely: in virtue of what does a specific (type of) mathematical structure apply to a given (type of) target system? In this vein one can ask, for instance, in virtue of what the natural number structure applies to the rabbit population in the example above; in virtue of what manifolds apply to spacetime; in virtue of what Hilbert spaces apply to quantum systems; and so on. We call this the special application problem. The specific problem is comparative: for a given (type of) target system what makes one type of mathematical structure apply to it rather than another? Should the rabbit population be embedded into the natural numbers? The rational numbers? The real numbers? What sort of function(s) should correspond to their physical interbreeding relations? Should they be bounded?

\footnotetext{
${ }^{5}$ Generating a strictly speaking false claim does not imply that the application is completely unsuccessful. Falsity comes in degrees, and so does being an unsuccessful application. There is clearly a sense in which some applications of mathematics generate claims about systems that are only approximately true (e.g. Newton's use of calculus to describe planetary motions), and yet one would not want to dismiss them as a unsuccessful tout court. However, quantifying degrees of success (or lack thereof) is tantamount to giving degrees of approximate truth, which is a problem we cannot tackle in this paper. For a review see Oddie's (2016). We would like to thank an anonymous referee for drawing our attention to this issue.

${ }^{6}$ Pincock (2012, Chapter 7) provides numerous more realistic examples of failed applications.
} 
And so on. There are multiple decisions to be made when determining which mathematical structure should be applied to a system in question, and how.

There is a third problem that is often mentioned in connection with the applicability of mathematics: in virtue of what, if at all, do facts about mathematical structures explain physical facts? We call this the explanation problem. This problem is of interest for at least two reasons. Firstly, philosophers of science are interested in what scientific explanation is and the question of how, if at all, mathematics can function explanatory has implications for this broader debate. Second, the recent literature on indispensability arguments for a commitment to the existence of mathematical objects has focussed explicitly on whether or not mathematics plays an essential explanatory role, rather than merely featuring in our "best scientific theories" as per the original QuinePutnam formulation (for a clear statement of this see Baker's (2009, Section 2)).

It is worth noting that the application problem, even if phrased in terms of successful application, is conceptually prior to the explanation problem. Before asking whether or not a fact about a mathematical structure explains some fact about a physical system we need to know whether or not the mathematical structure applies to the system in question. The examples in the recent literature - e.g. whether or not facts about prime numbers are essential in explaining the life cycles of cicadas (Baker 2005); whether or not the Borsuk-Ulam theorem explains the fact that there are two antipodal points on the Earth's surface with the same temperature and barometric pressure (Colyvan 2001); or whether or not the Honeycomb conjecture explains why bees build hexagonal honeycombs (Lyon and Colyvan 2008) - all presuppose that the relevant mathematical structures apply, and indeed apply successfully, to the physical system of interest. It's then an additional question as to whether or not the facts about the structures explain facts about the systems.

In this paper our focus is on the general applicability problem. We only make a few remarks about the specific problem on the way and briefly touch on the explanation problem in Section 6 where we indicate how our answer to the general application problem impacts on the explanation problem.

\section{The Mapping Account}

The core idea of what has become known as the mapping account is that a mathematical structure applies to a target system in virtue of there being a proposed structure-preserving mapping from the target system to the mathematical structure. The mathematical structure successfully applies to the target system if the mapping holds. Phrased in this way it's important to note that the proposal of the mapping answers the application question and whether the mapping holds adjudicates whether the application is successful.

The mapping account is phrased in terms of mathematical structures and it's 
important to be explicit about the notion of structure this presupposes. ${ }^{7}$ Structures are taken to be set-theoretic structures as used in logic and model theory. A structure in that sense is a composite entity consisting of a nonempty set $U$ of objects called the domain (or universe) of the structure and a nonempty indexed set $R$ of relations $r$ on $U$. It is convenient to write the structure as an ordered tuple $S=\langle U, R\rangle{ }^{8}$ For what follows it is important to be clear on the nature of the objects and relations that make up a set-theoretic structure. The important point is that it does not matter what the objects are intrinsically. The only thing that matters from a structural point of view is that there are so and so many of them. All we need are dummies or placeholders. Likewise for relations. It is irrelevant what the relation "in itself" is. All that matters from a structural point of view is between which objects it holds. For this reason, a relation is specified purely extensionally, that is, as class of ordered $n$-tuples and the relation is assumed to be nothing over and above this class. Thus understood, relations have no properties other than those that derive from this extensional characterization, such as transitivity, reflexivity, and symmetry.

Assume now that we want to apply the structure $S_{M}$ to a target system $T$. On the mapping account this involves proposing a mapping $f: T \rightarrow S_{M}$ such that $f$ preserves at least some of the structure of $T$. We then say that $S_{M}$ is the structure to be applied and that $f$ is the mapping that anchors the application of $S_{M}$. The nature of $f$ has been the subject matter of considerable debate with isomorphism, isomorphic embedding, partial isomorphism, and homomorphism being the most common suggestions. ${ }^{9}$ We say that $S_{M}$ and $T$ are morphic if the proposed morphism relating them exists.

This account of the applicability of mathematics is arguably implicit in many versions of the semantic view of theories, most notably in Suppes', van Fraassen's, the partial structures approach championed by Da Costa, French, Ladyman, and Bueno, and the Munich brand of structuralism due to Sneed, Stegmüller, Balzer and Moulines. ${ }^{10}$ Recent explicit statements and defences of the account have been offered by Pincock $(2007,2004,2012)$ and Bueno and Colyvan (2011). For our current purposes we focus on Bueno and Colyvan's discussion because their statement of the view is particularly helpful for a discussion of the issues we are interested in. ${ }^{11}$

\footnotetext{
${ }^{7}$ Explaining the application of mathematics by appeal to structures looks most natural in the context of structuralist philosophy of mathematics such as Shaprio's (1997) or Resnik's (1997). However, the mapping account does not presuppose this approach to mathematics and could be adopted by proponents of other accounts. We briefly return to this issue in Section 6.

8 The standard notion of a structure is introduced, for instance, in Hodges' (1997) and Enderton's (1972/2001). We note, however, that in the context of logic a structure is sometimes also taken to include a language and an interpretation function, which are absent from the notion of a structure as used in the current context.

${ }^{9}$ For a discussion of these see, for instance, Suppes' (1960), van Fraassen's (1980, 2008), Da Costa and French's (2003), French and Ladyman's (1999), Bueno's (2011), Bartels' (2006), Mundy's (1986), Pero and Suárez's (2016).

${ }^{10}$ For a discussion of these approaches see Portides' (2017).

${ }^{11}$ It's worth noting here that Bueno and Colyvan call their account an "inferential account" of the application of mathematics rather than a "mapping account". Whilst it's true that their account is
} 
Bueno and Colyvan break down the application of mathematics into three steps (ibid., p. 353):

(1) Immersion: "the first step consists in establishing a mapping from the empirical set up to a convenient mathematical structure [...] several mappings can do the job here, and the choice of mapping is a contextual matter, largely dependent on the particular details of the application."

(2) Derivation: "this step consists in drawing consequences from the mathematical formalism, using the mathematical structure obtained in the immersion step [...] This is, of course, the key point of the application process, where consequences from the mathematical formalism are generated."

(3) Interpretation: "finally we interpret the mathematical consequences that were obtained in the derivation step in terms of the initial empirical set up [...] To establish an interpretation, a mapping from the mathematical structure to the initial empirical set up is needed. This mapping need not be simply the inverse of the mapping used in the immersion step-although, in some instances, this may well be the case. But, in some contexts, we may have a different mapping from the one that was used in the immersion step."

In separating these three steps, Bueno and Colyvan elaborate on a simple mapping account in various important ways. Firstly, they are explicit that the choice of a mapping in the immersion step is a contextual matter and therefore does not require that the same mapping (let alone isomorphism) be used in every instance of applying mathematics. This, combined with the fact that they allow for distinct mappings from, and to, the empirical set up allows for instances where the mathematical structure contains more structure than the empirical one, and visa versa (ibid, p. 356). ${ }^{12}$ Secondly, they argue that after the immsersion step their account is flexible enough to accommodate multiple further mappings between the intitial mathematical structure and some further structures, and then applying the further two steps (ibid, p. 354). Thirdly, they argue that their account can accommodate idealisations via the introduction of partial mappings (ibid, pp. 356-363). Forthly, the argue that by allowing for pragmatic and contextual considerations to determine which mapping to choose on a case by case basis they make room for cases where novel predictions are generated by applying mathematics. This happened, for instance, where Dirac choose to interpet negative solutions to the dirac equation as physically meaningful (ibid, pp. 364-365). (They also claim that their account allows for mathematics to play an explanatory role, we return to this in Section 6).

Which of the two application problems introduced in Section 2 does this account address? Answers to the special problem are, by their very nature, contextual and no general account will, on its own, offer a solution to the question of how a

much richer than the simple version of the mapping account defined above, it does rely crucially on mappings to and from target systems and mathematical structures, and in this sense is an advanced version of, rather than an alternative to, the mapping account.

${ }^{12}$ A related point is made by Weisberg $(2013$, Section 3.3$)$ who points out that in cases where mathematical structures are used to represent a physical system, an assignment and intended scope are needed to specify which parts of the structure are mapped to which parts of the target system. Where the structure contains parts that don't correspond to any purported part of the target system (e.g. where irrational numbers satisfy the Lotka-Volterra equations) it should be made explicit that this is not supposed to have physical import. 
particular structure is applied (say, of how partial differential equations are applied to diffusion processes). The best a general account can do is offer a framework for the analysis of specific applications. Bueno and Colyvan's account does exactly that. As they point out, the inherent flexibility and context sensitivity of their framework allows them to account for Diarc's discovery of the positron; why we ignore negative solutions to quadratic equations in cases of predicating where a projectile will land; and the introduction of partial homomorphisms can, at least to some extent, accommodate cases like Simon's account of bounded rationality where the mathematical structures captures only some of the relevant features of the target system.

However, despite offering a framework in which to think about specific applications of mathematics, no complete answer to the general problem emerges from Bueno and Colyvan's account. The general problem is addressed in the first and the third steps when mappings between the empirical set up and convenient mathematical structures are established: every application of mathematics involves setting up at least two relevant mappings. But target systems are physical objects - atoms, planets, populations of rabbits, economic agents, and so on - and not structures. Yet a mapping $f$ is a relation that holds between two structures and claiming that a set theoretic structure is morphic to a piece of the physical world is prima facie a category mistake. If we are to make sense of the claim that the model is morphic to its target we have to assume that the target somehow exhibits a certain structure. Hence, formulated properly, applying structure $S_{M}$ requires defining a mapping $f: S_{T} \rightarrow S_{M}$ (and not $f: T \rightarrow S_{M}$ ) where $S_{T}$ is the structure of the target. But what is the relation between $T$ and $S_{T}$ ? What does it mean for a target system - a part of the physical world - to possess a structure? The answer to this question is far from obvious and Bueno and Colyvan are aware of this problem:

\footnotetext{
"Put simply, the world does not come equipped with a set of objects (or nodes or positions) and sets of relations on those. These are either constructs of our theories of the world or identified by our theories of the world. Even if there is some privileged way of carving up the world into objects and relations [...], such a carving, it would seem, is delivered by our theories, not by the world itself. What we require for the mapping account to get started is something like a pre-theoretic structure of the world (or at least a pre-modeling structure of the world). This is clearly a problem for the mapping account [...] the mapping account does require having what we shall call an assumed structure in order to get started. There is no avoiding such an assumption" (2011, p. 347).
}

Later in their paper, when discussing what makes their account more attractive than a simple mapping account, they argue that their account has the means to address this problem:

"the inferential conception is well placed to help provide the crucial assumed structure. Recall that the assumed structure is the structure the modeling exercise assumes to be present in the world [...] we will need to impose some structure on the world in order to begin the modeling exercise. Earlier we suggested that the mapping account might treat this initial assumed structure as defeasible and let the resulting mathematical model help inform refinements or revisions to the initial assumed structure. The inferential account has the resources to make revisions midstream and does not require starting from scratch each time a more fruitful assumed structure is conceived. This is achieved 
by employing the composite mappings to move from the mathematized initial assumed structure to another mathematical structure, where the latter may be thought to correspond to a new (revised) assumed structure. There is no need to revise formally the initial assumed structure because the interpretation step of the process will deliver the final structure of the empirical set up-one informed by the modeling exercise and one that may well be quite different from the initial assumed structure" (2011, p. 357).

Bueno and Colyvan are right in pointing out that their account offers increased flexibility, but this does not explain how a structure emerges from something non-structural in the first place. To revise a structure presupposes that there is a structure to begin with, but Bueno and Colyvan have little to say about where the "assumed structure" comes from. Likewise, Pincock signals awareness of there being "an important difference between talking about a concrete system made up of objects and linked together by concrete relations involving quantities and properties and a set-theoretic structure" (2012, p.29), but then says little about how this gap is to be bridged. Hence, that there is a structure to begin with remains an unanalysed posit, and with it the general application problem is left unresolved.

\section{The Curse of Abundance}

In order to make sense of the mapping account, an account needs to be given of where the structure of a target system comes from. In this section we review existing accounts and argue that they are dead ends.

A first suggestion is that data models are the sought-after "assumed structure" of the target system. Mathematics is not applied to a mysterious structure of the system itself, but to a data model that results from performing measurements on the system. The idea that data might provide the required target-end structure originated in a different context, namely the issue of representation in the socalled semantic view of theories. The idea is originally found in Suppes' (1962/1969), but has been most developed by van Fraassen (2008). ${ }^{13}$ Experimental measuring processes gather raw data. These are then cleaned, with anomalous data points rejected and measurement error taken into account. Then they are often idealized, for instance by replacing discrete points by a continuous curve. Where these data points are numeric, the smooth curve is a function that can be treated as a relation defined over $\mathbb{R}$, or $\mathbb{R}^{\mathrm{n}}$, or intervals thereof. These data models are set theoretic structures, and therefore they can enter into the morphisms as required by the mapping account.

The main problem facing this idea is that if this is the end of the story then it turns out that mathematics doesn't apply to the physical world after all because data themselves are mathematical objects - in fact this is what allows them to play the required role of the "assumed structure" in the mapping account. So the account ends up applying mathematics to mathematics, and hence begs the question of how mathematics is applied to the world. In the context of scientific

\footnotetext{
13 For a critical discussion (that pre-dates van Fraassen's 2008 development) see Brading and Landry's (2006).
} 
representation van Fraassen (2008) responds to this objection by invoking pragmatic aspects of the context of using scientific models. In the language of the mapping account, the core of van Fraassen's argument is that there is no pragmatic difference between taking a mathematical structure to apply accurately to a physical system and accurately apply to data extracted from it. Nguyen (2016) provides a critical discussion of van Fraassen's argument in the context of theory of representation and concludes that the argument is unsuccessful in deflating the gap between data and physical systems. Nothing in Nguyen's argument depends on whether the problem at hand is representation or the applicability of mathematics, and hence the argument carries over mutatis mutandis. But in the absence of a successful argument deflating the gap between data extracted from a system and the system itself, appealing to data to provide the structure of a physical systems delivers an account of the applicability of mathematics whereby mathematics applies to other parts of mathematics, rather than the physical world proper.

The second suggestion of where to find the "assumed structure" of a physical system is more radical. One could simply claim that physical systems themselves are structures. Prime facie this seems like a category mistake: structures are sets with relations and functions existentially defined upon them, and physical systems are not sets. However Tegmark (2008) argues the contrary. His argument proceeds from what looks like an innocuous claim: there exists an external physical reality completely independent of us humans (what he calls the "external reality hypothesis"). He argues that this entails that the world is a mathematical structure (his "mathematical universe hypothesis"). His argument for this is based on the notion that a so called "theory-of-everything" must be expressible in a form that is devoid of human-centric "baggage" (by the external reality hypothesis), and the only theories that are devoid of such baggage are mathematical, which, strictly speaking, describe mathematical structures. Thus, since a complete theory of everything describes an external reality independent of humans, and since it describes a mathematical structure, the external reality itself is a mathematical structure.

This approach stands or falls on the strengths of its premise that a complete theory of everything will be formulated purely mathematically, without any human baggage, which in turn relies on a strict reductionist account of scientific knowledge. We have doubts about a number of steps in this argument, in particular the validity of inference from the proposition that a theory of everything describes a mathematical structure to the conclusion that the external reality itself is a mathematical structure. However, an in-depth assessment of Tegmark's argument is beyond the scope of this paper. For our current purposes is sufficient to note that Tegmark's discussion is focused on the claim that fundamentally the world is a mathematical structure. Even if this is the case, the argument fails to illuminate any case where mathematics is applied to a target system at the non-fundamental level. Arithmetic can be used to count objects on the desk; a biologist might use the Lotka-Volterra equations to generate a prediction about a predator-prey system; an economist might use a pair of utility and probability functions that map to the real numbers to summarise someone's choice behaviour; and so on. It is undeniable that these 
are instances of applying mathematics. Yet these target systems are not a fundamental part of the world, nor do models of these objects have to make reference to their fundamental constituents. When modelling an airplane wing, aeronautic engineers don't first identify the fundamental super-string structure of the bits of matter that make up the wing and then construct wing models that are isomorphic to such fundamental structures. So even if Tegmark's account is correct about the fundamental level (and there is serious "if" about this), it offers no answer to the question about where structures are to be found at the level of non-fundamental target systems.

If data don't provide a suitable proxy for the structure of a target system, and a target system shouldn't be identified with such a structure, then what other options are available? We take it that the idea that either implicitly or explicitly underlies the mapping account is that target systems instantiate structures, where the instantiation relation that holds between a target system and a structure is supposed to be the sort of relation that holds between an object and a property, or a pair of objects and a relation they enter into. This comes across most clearly when structuralists in the philosophy of mathematics discuss the relationship between mathematics and the physical world. For example, Shapiro argues that:

"[On the structuralist account of mathematics] the problem of the relationship between mathematics and reality is a special case of the problem of the instantiation of universals. Mathematics is to reality as universal is to instantiated particular. As above, the 'universal' here refers to a pattern or structure; the 'particular' refers not to an individual object, but to a system of related objects. More specifically, then, mathematics is to reality as pattern is to patterned" (1983, p. 538). ${ }^{14}$

Let us then assume that a structure $S$ defines a universal. The main question then is: under what conditions does a target instantiate a certain structure $S$ ? The details here might depend on which account of universals one adopts, but what all answers would seem to have in common is the following. Since a structure consists of a set of objects with relations on them, the target $T$ instantiates structure $S_{T}$ iff $T$ consists of individuals that make up the domain of $S_{T}$ and enter into the relations that are specified in $R$.

Natural as this may sound, this definition faces an immediate problem. The problem is Newman's objection to Russell's structural realism (Newman 1928). The essential point of the objection is that for any structure $S=\langle U, R\rangle$ where the cardinality of $U$ is $k$, a target system consisting of $k$ objects instantiates a

\footnotetext{
${ }^{14}$ A similar position is found in Resnik's (1997, p. 204). Due to the fact that some mathematical structures are not instantiated by any physical system, Shapiro further distinguishes between whether or not structures as universals should be thought of as ante rem universals, in re universals (eliminative structuralism) or in modal terms (Shapiro 1997). These distinctions are immaterial to our question. A word of warning about terminology is in order here. Shapiro often uses the term "exemplified" to refer to the relationship between a universal and a physical system. We prefer "instantiates" given that that "exemplification" is used in a slightly different way in the literature of representation following Goodman and Elgin, and has been incorporated into the literature on scientific representation in our (forthcoming).
} 
structure isomorphic to $S$ in the sense that it is possible to extensionally define relations matching the relations in $R$ in terms of $n$-tuples of the objects in the target system. ${ }^{15}$ So if we think about instantiation in this way, then we are left with the fact that a target system instantiates any structure that has the cardinality of the target.

We are now faced with an identification problem. Recall Bueno and Colyvan's first step, immersion, which consists in "establishing a mapping from the empirical set up to a convenient mathematical structure" (op cit.). To establish a mapping it is not enough to show that there exists some structure $S_{T}$ in the target such that there exists some mapping $f$ from $S_{T}$ to $S_{M}$. Applying a certain structure $S_{M}$ requires, as Bueno and Colyvan aptly put it, establishing a mapping. This, in turn, involves identifying a particular structure $S_{T}$ in the target and constructing a mapping $f$ from that structure to $S_{M}$, the structure that is to be applied. Or to put it another way, applying $S_{M}$ is an agent-based notion - it's something that a scientist does (which ties in with Bueno and Colyvan's emphasis on pragmatic factors). To apply a structure $S_{M}$ to a target the scientist has to single out a particular target structure and explicitly construct the mapping $f$ by matching up elements in the domains of the structures and pairing up relations. If there was only one structure in target - if there was such a thing as "the" structure of the target - then there was no question about this choice and the remaining issue would only be how exactly the scientist gains access to that structure. But faced with the Newmanian abundance of structures, the scientist wanting to apply maths is faced with serious underdetermination problem: which of the many structures should she pick and how?

One might argue that Newman's objection is just logical trickery and once a few sensible assumptions about the system are made the underdetermination is resolved. This is in effect the route taken by the standard ways of blocking Newman's objection, which involve appeal to natural kinds in one way or other. ${ }^{16}$ One may have all kind of quibbles about natural kinds, but let us set these aside for the sake of argument. It is our contention that even if natural kinds are assumed to be unproblematic, an appeal to them is not sufficient to resolve the remaining underdetermination issues. Let us illustrate this point with an example, the methane molecule. ${ }^{17}$ The molecule consists of a carbon atom and four hydrogen atoms grouped around it, forming a tetrahedron. Between each hydrogen atom and the carbon atom there is a covalent bond.

\footnotetext{
${ }^{15}$ For a detailed discussion of the theorem see Ketland's (2004). However, the basic point is straightforward and can shown as follows. Let $\langle U, R\rangle$ be a structure such that $|U|=k$. Let $T=\left\{x_{1}, \ldots, x_{k}\right\}$ be a set consisting of the $k$ objects from the target system. Since $|T|=|U|=k$ there is a bijection $f: T \rightarrow U$. Using this bijection, for each $r_{i} \in R$, we can construct a set $r^{\prime}$ consisting of $n$-tuples of objects from $T$ as follows: $\left\{\left\langle x_{i}, \ldots, x_{j}\right\rangle:\left\langle f\left(x_{i}\right), \ldots, f\left(x_{j}\right)\right\rangle \in r_{i}\right\}$. Collecting these relations together gives a structure, $\left\langle T, R^{\prime}\right\rangle$ which is isomorphic to $\langle U, R\rangle$ by construction.

${ }^{16}$ For a discussion of the various responses see Ainsworth's (2009).

17 What follows is a variation on an example discussed in Frigg's (2006, pp.57-58).
} 
Carbon, hydrogen and covalent bonds are bona fide natural kinds. Describing the molecule in these terms does, however, not fix a structure uniquely. One can regard the atoms as objects and the bonds are relations. Denoting the carbon atom by $a$, and the four hydrogen atoms by $b, c, d$, and $e$, we obtain a structure $S_{T}$ with the domain $U=\{a, b, c, d, e\}$ and the relation $r=\{\langle a, b\rangle,\langle b, a\rangle,\langle a, c\rangle$, $\langle c, a\rangle,\langle a, d\rangle,\langle d, a\rangle,\langle a, e\rangle,\langle e, a\rangle\}$, which can be interpreted as 'being connected by a covalent bond'. Alternatively, however, one could just as well choose bonds as objects and consider the relation 'sharing a node with another bond'. Denoting the bonds by $a^{\prime}, b^{\prime}, c^{\prime}$ and $d^{\prime}$, we obtain a structure $S_{T}{ }_{T}$ with the domain $U^{\prime}=\left\{a^{\prime}, b^{\prime}, c^{\prime}, d^{\prime}\right\}$ and the relation $r^{\prime}=\left\{\left\langle a^{\prime}, b^{\prime}\right\rangle,\left\langle b^{\prime}, a^{\prime}\right\rangle,\left\langle a^{\prime}, c^{\prime}\right\rangle\right.$, $\left\langle c^{\prime}, a^{\prime}\right\rangle,\left\langle a^{\prime}, d^{\prime}\right\rangle,\left\langle d^{\prime}, a^{\prime}\right\rangle,\left\langle b^{\prime}, c^{\prime}\right\rangle,\left\langle c^{\prime}, b^{\prime}\right\rangle,\left\langle b^{\prime}, d^{\prime}\right\rangle,\left\langle d^{\prime}, b^{\prime}\right\rangle,\left\langle c^{\prime}, d^{\prime}\right\rangle$, $\left.\left\langle d^{\prime}, c^{\prime}\right\rangle\right\}$. Obviously $S_{T}$ and $S^{\prime}{ }_{T}$ are not isomorphic. So which structure is picked out depends on how the system is described. Depending on which parts one regards as individuals and what relation one chooses, very different structures can emerge. And it takes little ingenuity to come up with further descriptions of the methane molecule, which lead to yet other structures.

There is nothing special about the methane molecule, and similar stories can be told about any target. Consider again the example of applying mathematics to the rabbit population mentioned in Section 2. When faced with the population there are any number of ways in which the system considered as a whole could be carved up. Regarding the objects in the system the scientist could count individual rabbits as above. Or she could take pairs of rabbits as the relevant objects. Or she could count rabbit legs (perhaps she is interested in selling them as lucky charms). Or she could count rabbit hairs, or rabbit teeth. Or she could take individual molecules in the system to form the basic objects and define elaborate structures on a massive set containing them. For any target system the question of what to take as the objects and the relations is a substantive question inviting multiple answers. ${ }^{18}$ And even once the objects are fixed, there is no unique answer as to what relations between them should be considered. The scientist could care about the reproduction behaviour of the rabbits, or different ways of grouping them according to their fur, or grouping them according to the length of their ears, and so on. So the lesson learned generalises: there is no such thing as "the" structure of a target system. There are any number of ways of picking out a set of objects to form the domain of a structure of the target system. And even once this is done, any number of different physical properties and relations that might be of interest would deliver a different structure to which the mathematical structure would be applied via a proposed morphism.

In their description of the "immersion" step Bueno and Colyvan identify theories as the actors that provide a structure. They say that the elements of a structure

\footnotetext{
${ }^{18}$ Weisberg (2013, Section 5.3.1) makes a similar point when he draws a distinction between phenomena and target systems. He claims that the former stand in a one-to-many relationship with the latter, where a model's "intended scope" specifies a (proper) subset (target system) of the total state of the system (phenomena). Having said that, our point here goes beyond his: even once a target system of interest is fixed, there still remains the question concerning its structure (cf. the example of the methane molecule which would seem to count as a target system on any reasonable reading of the term).
} 
are "constructs of our theories", which deliver "some privileged way of carving up the world into objects and relations" (op. cit). This suggestion, however, runs into the same difficulties as natural kinds. While theories may play a role in identifying a structure, they do not determine a unique structure. Newtonian mechanics, for instance, instructs us to identify objects and the forces acting on them, but it doesn't prescribe how exactly this has to be done. Just like a population of rabbits, a galaxy of stellar bodies can be carved up into objects and relations in many different ways.

So we are still faced with the above identification problem. If the relationship between target systems and their structures is not one of brute metaphysical fact, and if the same target system has multiple structures, then when someone proposes a structure-preserving mapping between a target structure and a mathematical structure, how are we to determine which of those target structures are being invoked? Without an answer to this question it's not clear either that the invoked structure preserving mapping is well defined, or if it is, then whether or not the application of mathematics is successful.

From this we don't conclude that Bueno and Colyvan's account is wrong, but that it is incomplete. "Immersion" is a metaphor that needs to be unpacked. Their presentation seems to suggest that once a theoretical background is assumed a relevant structure emerges in a relatively unproblematic manner, and those interested in the applicability of mathematics can take this structure for granted without probing further into its nature and origins. ${ }^{19}$ The considerations in this section show that there is more to the problem of the attribution of structure than meets the eye at first glance. There is a serious issue where the initial structure $S_{T}$ comes from, and the issue is not merely one of philosophical housekeeping. Without further explanations, we neither know what $S_{T}$ is, nor are we able to specify the proposed morphism that anchors the application of $S_{M}$.

\section{A Positive Proposal}

In this section we introduce what we call the extensional abstraction account of generating a target structure. In essence, we propose that for the mapping account to get off the ground, a scientist has to describe the target system in physical terms, and then by abstracting away to the extension of such a description, generate a target structure to anchor an application of mathematics.

The solution to the problem we have been addressing follows from a careful analysis of the above cases. Recall the scenario that the scientist is faced with when applying mathematics to a population of rabbits. We described different ways she might do this, ways that involved selecting different parts of the system as objects and identifying different physical properties and relations as relevant depending on her purpose at hand. In doing so she provided what we call

\footnotetext{
${ }^{19}$ See Bueno and Colyvan's (2011, p. 347) quoted above. As discussed, Bueno and Colyvan allow for revisions of the "assumed structure" downstream. But our concern is prior: where did the original structure come from in the first place?
} 
different structure generating descriptions of the same system in each instance. In general a structure generating description does three things:

(1) It describes certain aspects of the system as the relevant objects. In the above example different descriptions described individual rabbits, rabbit legs, or molecules that make up the rabbits as the relevant objects of the system.

(2) It describes certain aspects of the system as relevant physical properties and relations. In the above examples these were physical relations that held between interbreeding rabbits, descriptions of the rabbits' fur, and so on.

(3) It describes the objects described in (1) as instantiating the physical properties and relations described in (2).

In general a structure generating description $\boldsymbol{D}_{\boldsymbol{s}}$ has the form: "the target system $T$ contains objects $\boldsymbol{o}_{1}, \ldots, \boldsymbol{o}_{n}$ which either individually, or in collections, instantiate physical properties and relations $\boldsymbol{r}_{\mathbf{1}}, \ldots, \boldsymbol{r}_{\boldsymbol{m}}$." It's important to note that $\boldsymbol{D}_{\boldsymbol{s}}$ contains terms that describe the system in physical, not set-theoretic terms, and we use bold font to indicate that the terms in $\boldsymbol{D}_{\boldsymbol{s}}$ refer to physical properties and relations. The $\boldsymbol{o}_{\boldsymbol{s}}$ are terms like "rabbit" and "rabbit leg" and not "elements of a set". Likewise, the $\boldsymbol{r}_{\boldsymbol{s}}$ are terms like "breeding with" or "being larger than" and not "being an $n$-tuple".

With this in mind it now transpires how a structure generating description earns its name and actually generates a structure for a target system for the mapping account to get a grip on. Recall from Section 3 that a set-theoretic structure consists of featureless dummy-objects that enter into purely extensionally defined relations. $\boldsymbol{D}_{\boldsymbol{s}}$ generates such a structure if we remove the physical nature of the objects and relations from $\boldsymbol{D}_{\boldsymbol{s}}$. In the case of the $\boldsymbol{o}_{s}$ this means that we move from describing the system as consisting of rabbits to describing it as consisting of objects. If $\boldsymbol{D}_{\boldsymbol{s}}$ talks of, say, five rabbits (and pinpoints them by picking out a particular rabbit as the first rabbit, another one as the second rabbit, etc.), we now remove "rabbits" from this description and only keep "objects" (and, possibly, a numbering of them as the first object, the second object, etc.). The relations get stripped of their physical nature by replacing them with their extension. If $\boldsymbol{D}_{\boldsymbol{s}}$ describes the relation as "breeding with", we now remove "breeding with" and only keep the extension of the relation, which only specifies between which objects the relation holds but not what the relation itself is. We call the resulting description $D_{s}$ (now no longer in bold font). While $\boldsymbol{D}_{\boldsymbol{s}}$ describes $T$ as consisting of interbreeding rabbits, $D_{s}$ describes it as consisting of so-and-so many objects that enter into purely extensionally defined relations. $D_{s}$ is a purely structural description, which specifies a structure $S_{T}$ consisting of $n$ dummyobjects $o_{1}, \ldots, o_{n}$ which enter into purely extensionally defined relations $r_{1}, \ldots, r_{m}$ (note that symbols are now no longer in bold font). This structure is what enters into the mapping relation required by the mapping account. 
The transition from $\boldsymbol{D}_{\boldsymbol{s}}$ to $D_{s}$ can be described as a process of abstraction, hence the name "extensional abstraction account". ${ }^{20}$ We start with a "thick" description $\boldsymbol{D}_{\boldsymbol{s}}$ of the target and then, by walking up the ladder of abstraction, strip away all material properties of the object until we are left with objects that are nothing but objects, and relations that are nothing but $n$-tuples of nothing-but-objects. But unlike Wittgensteinian ladders, which can be thrown away once we've reached the top, abstraction ladders have to remain in place. First, $\boldsymbol{D}_{\boldsymbol{s}}$ specifies to what the structure is applied. The description $\boldsymbol{D}_{\boldsymbol{s}}$ anchors the application of structure $S_{T}$ to the target, and without $\boldsymbol{D}_{\boldsymbol{s}}$ the application has been lost because if we remove $\boldsymbol{D}_{\boldsymbol{s}}$ from the picture we are left with nothing but an abstract structure. Second, Bueno and Colyvan's third step requires us to "interpret the mathematical consequences that were obtained in the derivation step in terms of the initial empirical set up" (op. cit). This is impossible without $\boldsymbol{D}_{\boldsymbol{s}}$ (or at least some description of the target system in physical terms). If we throw the ladder away, we cannot climb down any more and bring mathematical results back to the domain of application. For these reasons a structure $S_{T}$ applies to target $T$ always only relative to a structure generating description $\boldsymbol{D}_{\boldsymbol{s}}$, and the phrase of "applying $S$ to $T$ " should be seen as an ellipse for "applying $S$ to $T$ relative to the structure generating description $\boldsymbol{D}_{\boldsymbol{s}}$ ". Without $\boldsymbol{D}_{\boldsymbol{s}}$ the claim that a structure $S$ applies to $T$ is meaningless. For this reason mathematics is not the only language in the book of nature!

One might ask when in the process we have outlined we enter the realm of mathematics: is it when we transition from $\boldsymbol{D}_{\boldsymbol{s}}$ to $D_{s}$ (and with it to $S_{T}$ ), or is it when $S_{T}$ is connected to $S_{M}$ through a suitable morphism? The answer to this question depends on how strictly one characterises "mathematics", and where one puts the emphasis. If one takes any use of set theory to be mathematics, then the application happens in the transition from $\boldsymbol{D}_{\boldsymbol{s}}$, via $D_{S,}$, to $S_{T}$. This seems to be suggested, for example, by Malament (1982, pp. 529-532) in his discussion of whether Field (1980) is entitled to use sets of spacetime points in his "nominalist" reconstruction of Newtonian gravity. Under a stricter reading of "mathematics", where one asks at what point the sorts of structures studied by mathematicians (e.g. those that appear in differential geometry or linear algebra) enter the picture, application happens when one proposes to connect $S_{T}$ to $S_{M}$ through a suitable morphism. We think that this is the sense of "application" that most proponents of the mapping account have in mind. We take it as an attribute of our approach that it can distinguish between these subtlety different senses of the application of mathematics. Ultimately both senses are legitimate and a choice between them depends on one's interest and other philosophical commitments.

So our answer to the general application problem is the following. A mathematical structure $S_{M}$ applies to a target $T$ iff a structure generating description $\boldsymbol{D}_{\boldsymbol{s}}$ of $T$ is given which, through a process of abstraction, is turned into a purely extensional description $D_{s}$ that describes a structure $S_{T}$, which is then proposed to be appropriately morphic to $S_{M}$. With this in place, the details of the

\footnotetext{
${ }^{20}$ Abstraction can be explicated a number of different ways. One way that we find conducive is Cartwright's (1999, Ch. 3), but nothing in what follows depends on what account of abstraction one adopts.
} 
morphism between $S_{M}$ and $S_{T}$ (and possibly further mathematical structures) can be spelled out on a case-by-case basis by the mapping account's answer to the special applicability problem. As such, once $\boldsymbol{D}_{\boldsymbol{s}}$ has done its job and generated $S_{T}$, all of the flexibility of, for instance, Bueno and Colyvan's more nuanced mapping account, which takes into account contextual features in specifying the appropriate morphism, can be accommodated. ${ }^{21}$

Our account, even though schooled on a simple population model, is in no way limited to this kind of application. An important place where mathematics is applied to the physical world is in fundamental measurement, and on closer inspection it turns out that representation theorems in the representational theory of measurement are a good place to see our approach in action. ${ }^{22}$ Consider a simple example where the system in question is a collection of rods. The scientist can describe the system in a way that takes the individual rods to be the objects in the system, and a length comparison between them to be the relevant physical relation. This way of looking at the system provides the following structure generating description:

$\boldsymbol{D}_{\text {l: }}$ "The target system consists of $n$ rods $\boldsymbol{o}_{1}, \ldots, \boldsymbol{o}_{\boldsymbol{n}}$. The rods enter into the comparative physical relation $\boldsymbol{r}=$ longer or equal in length than. It is the case that the rods have been labelled so any two consecutive rods stand in relation $\boldsymbol{r}: \boldsymbol{r}\left(\boldsymbol{o}_{2}\right.$, $\left.\boldsymbol{o}_{1}\right), \boldsymbol{r}\left(\boldsymbol{o}_{3}, \boldsymbol{o}_{2}\right), \ldots, \boldsymbol{r}\left(\boldsymbol{o}_{n}, \boldsymbol{o}_{n-1}\right)$. It is also the case that if $\boldsymbol{r}\left(\boldsymbol{o}_{m}, \boldsymbol{o}_{m-1}\right)$, then $\boldsymbol{r}\left(\boldsymbol{o}_{m}, \boldsymbol{o}_{k}\right)$ for all $k=1, \ldots, m-1$ and for all $m=2, \ldots, n . " 23$

This description is now subjected to the above process of abstraction, which yields:

$D_{l}$ : "There are $n$ object $o_{1}, \ldots o_{n}$ which form a set $U=\left\{o_{1}, \ldots, o_{n}\right\}$ and a relation $r$ on $U$ such that $r\left(o_{m}, o_{k}\right)$ for all $k=1, \ldots, m-1$ and for all $m=2, \ldots n . "$

This description specifies a structure $S_{T}$ with the domain $U=\left\{o_{1}, \ldots, o_{n}\right\}$ and the relation set $R=\{r\}$ of relations on $U$. Thus, $D_{l}$ generates $S_{T}=<U, R>$ via $D_{l}$.

This structure can now be embedded in other more elaborate structures. Different structures can be chosen, and different morphisms can be used. For instance, let $\langle\square, \geq\rangle$ be the set of the natural numbers with the relation $\geq$ (greater than or equal) defined on them. To apply the structure $\langle\square, \geq\rangle$ to the system of rods the scientist has to propose that there is a mapping $f: U \rightarrow N$ such that:

\footnotetext{
${ }^{21}$ In our example of the dynamics of a population this allows for embedding mappings between discrete structures like $S_{T}$ and continuous structures involving $\square$, as used, for example, in the Lotka-Volterra equations. These discrete-to-continuous mappings are not restricted to biology; see Maddy's (1995, pp. 254-255) for a nice discussion of Feynman's concerns that time could be discrete despite the fact continuous structures are used to represent it. We are grateful to Mark Colyvan for bringing this example to our attention.

22 The classic source is Krantz et. al's (1971). Brown (1999, Ch. 4) offers an introduction; Diez (1997a, 1997b) provides an overview of the development of the theory.

${ }^{23}$ Those who worry that labeling rods with numbers is already an application of mathematics and that $\boldsymbol{D}_{\mathbf{1}}$ therefore presupposes what it is supposed to provide can replace the names $\boldsymbol{o}_{\boldsymbol{k}}$ by ordinary proper names and name the rods "Jim", "Mina", "Emily", etc.
} 


$$
\text { for all } o_{x}, o_{y} \in U:\left\langle o_{x}, o_{y}\right\rangle \in r \leftrightarrow\left\langle f\left(o_{x}\right), f\left(o_{y}\right)\right\rangle \in \geq .
$$

If this condition is met by $f$, then we can say that the application of the natural numbers to the system of rods is successful and the scientist can use facts about the ordering of the natural numbers to derive (simple) hypothesis about the ordering of the rods in the system by length.

Much of the theory of fundamental measurement is concerned with the choice of the right structures and morphisms, and there are deep and interesting questions there. What matters in the present context is that constructing the structure $\langle U, R\rangle$ involved choices for the scientist at every step: a choice concerning the objects in the system; a choice concerning the relevant physical properties and relations; and a choice concerning how the former instantiate the latter. We are led astray if we think that $\langle U, R\rangle$ simply drops out of the system because it's somehow "natural". $\langle U, R\rangle$ may seem natural because there are significant choices available to the scientist "upstream" of the application of mathematics, choices that the description generating the structure respects. But this is an ex post facto judgement and countless alternative descriptions of the system are available, including, for instance, the following:

$\boldsymbol{D}_{\boldsymbol{t}}$ : "The target system contains rods $\boldsymbol{o}_{1}, \ldots, \boldsymbol{o}_{\boldsymbol{n}}$ and these enter into the relation $\boldsymbol{r}_{\boldsymbol{t}}$ = thicker than."

$\boldsymbol{D}_{\boldsymbol{m}}$ : "The target system contains rods $\boldsymbol{o}_{1}, \ldots, \boldsymbol{o}_{\boldsymbol{n}}$ and some have property of $\boldsymbol{r}_{\boldsymbol{m}}=$ being made out of mahogany and others $\boldsymbol{r}_{\boldsymbol{b}}=$ being made out of out of birch."

$\boldsymbol{D}_{\boldsymbol{c}}$ : "The target system contains molecules $\boldsymbol{o}_{1}, \ldots, \boldsymbol{o}_{\boldsymbol{m}}$, some of which have the property of being $\boldsymbol{r}_{\boldsymbol{w}}=$ being water, and others have the property $\boldsymbol{r}_{\boldsymbol{c}}=$ being cellulose."

Each of these descriptions generates a different structure. So, the system doesn't have a unique "natural" structure that the scientist somehow picks up. Rather, the scientist's particular purposes for applying mathematics are such that she has to make a decision regarding how to describe the physical system and then the description chosen provides a structure for her to work with when applying mathematics.

Three observations are in order. First, there is no requirement that the physical relations referred to in the structure generating description be operationalized. In some cases this may be so, but in others it may not. All that is required is that the relations be physical relations.

Second, there is also no expectation that we have access (either by direct acquaintance or through other sorts of observation) to the physical objects or relations described by $\boldsymbol{D}_{s}$. The elements of the structure can be hypothesized. We can ascribe a size to the rabbit population hundred years from now, and we can specify the distance between two atoms. This is particularly pertinent in cases of 
the application of mathematics in the more "fundamental" areas of physics. Here, there may be cases where the physical objects and relations are so far removed from our experience that it is difficult to see how we could have a good grasp of a description $\boldsymbol{D}_{\mathbf{s}}$ independent of the mathematics used to represent them.

Here it's important to emphasise the distinction between an agent being able to offer a description $\boldsymbol{D}_{\boldsymbol{s}}$, and that agent knowing $\boldsymbol{D}_{\boldsymbol{s}}$ to be true. Our account requires the former but not the latter - it suffices that such a description is given, regardless of whether it's known to be true or false. If $\boldsymbol{D}_{\boldsymbol{s}}$ also happens to be true, then the stage is set for a successful application of mathematics, assuming that the structure generated by the description is appropriately morphic with some proposed mathematical structure. We take it that many cases in fundamental physics take this form. The atoms in a crystal are located at a certain distance from each other; molecules are said to have certain size; particles in the standard model are described as having physically meaningful properties like mass and charge; and the purported fundamental constituents of the world are described as having the shape of strings.

Could there be cases where mathematics is "applied", and yet no physical description whatsoever is offered? Such cases could take one of two different forms: either such a description is assumed to exist in principle, even if we don't currently have access to it, or such a description is claimed not to exist at all. In the former case, if we knew that a physically rich description existed in principle then presumably we could in practice offer something like the following:

$\boldsymbol{D}_{\boldsymbol{x}}$ : "the target system contains some physical objects $\boldsymbol{x}_{1}, \ldots, \boldsymbol{x}_{\boldsymbol{n}}$ and some have some physical property $\boldsymbol{r}_{\mathbf{1}}=X_{1}$, and others the physical property $\boldsymbol{r}_{\mathbf{1}}=X_{2}$, and so on",

where ' $\boldsymbol{x}$ ' and ' $X$ ' are used to indicate that we have no way of describing what the physical objects or properties are.

Notice that such a description looks remarkably like a Ramsey-sentence, as associated with some versions of epistemic structural realism (for an overview see Frigg and Votsis' (2011)). On this view it's not simply that we don't know about the "nature" of the objects and relations in the world, but we cannot even describe them. In such cases we face a potential application of mathematics, which becomes an application proper once the physical descriptions are offered.

In the latter case no physical description is given and it is assumed that none exists, even in principle. In such a case it seems inappropriate to us to talk about "applying" mathematics to a target. Mathematics is used to generate claims about some system, but not in a way in which the mapping account is supposed to capture.

Third, we note that our take on the application of mathematics is consonant with certain prominent views about models and data. Hartmann (1999) and Morgan (2001) emphasise that models come accompanied by stories, and Muller (2011) reminds us that "all actual data structures float in sea of stories, that need to be 
told in order to know which data are relevant for which theory" (ibid., p. 100). These observations were not intended as contributions to a discussion of the general application problem. However, they are made in contexts where the use of mathematical structures in science is discussed and so they can be seen as evidence that, at least implicitly, a view very similar to ours is assumed in these discussions.

\section{Concluding Remarks}

By way of conclusion we want to make a few observations about how the view we developed in the last section relates to other issues in connection with the application of mathematics. Let us first return to the explanation problem that we introduced in Section 2 and then set aside. Bueno and Colyvan argue that simple versions of the mapping account have difficulty accounting for mathematical explanations. ${ }^{24}$ They argue as follows:

"If mathematics is genuinely explanatory, however, this will present a serious
problem for the mapping account. The problem is simply that it is hard to see
how a mere representational system can provide explanations and yet that is the
only role mathematics is allowed to play in the mapping account. [footnote
omitted] Consider once again our map of a city. Certain facts about the city will
be more obvious in the street map-indeed, that's the purpose of a street map-
but it would be very odd to think of the map as providing an explanation of any
facts about the city" (2011, p.351).

Bueno and Colyvan are right in pointing out that a representation does not ipso facto double as an explanation. This, however, does not preclude representations from playing a role in explanations. A representation can contain information that is explanatorily relevant. The London tube map, for instance, does not explain why one cannot get directly from Brixton to Stratford. But it contains information that is explanatorily relevant, namely that the two stations are not on the same line. In a similar vein, a mathematical structure may contain information that is explanatorily relevant even though the structure as such is not an explanation. Depending on one's account of explanation one will recognise different pieces of information as explanatorily: counterfactual dependence, law-like connections, causal relations, the use of unificatory patterns, and so on. Information of this kind can be encoded in a mathematical formalism, and by connecting the formalism to the target the mapping account makes this information relevant for a target system. In this way the mapping account is indeed an important ingredient in any mathematical explanation because without a mapping a mathematical formalism would be "free floating" and have no bearing on the target in question. Hence, while a structure that's mapped to target is not ipso facto an explanation, the existence of such a

\footnotetext{
${ }^{24}$ Recall that Bueno and Colyvan describe their account as an "inferential" conception of the application of mathematics, rather than a mapping account, and they take it that their account has the resources to account for mathematics playing an explanatory role. They take it that it is the choice of mapping at the interpretation and immersion stages that allows explanations to be obtained (2011, p. 366).
} 
mapping is a precondition for (at least some kinds of) explanation.

An issue that that arises in connection with the view developed in the last section is nominalism. By anchoring structures in physical descriptions, aren't we implicitly committing to nominalism? There are two observations that seem to pull into that direction. The first is that by anchoring structures in something physical we reduce structures to the physical and hence make them obsolete. This is too quick. Firstly, although the structure of a target may be anchored to a physical description, our account does not preclude the existence of structures not so anchored - the structures proposed to be appropriately morphic to a target structure do not in fact need to be anchored. Secondly, our discussion attempts to elucidate what it means for a target to have a certain structure, but this is metaphysically neutral. Consider the analogy with ordinary objects: that a table consists of atoms and that the atoms are arranged in certain way does not prove that the table is unreal. Of course some may want to draw this conclusion; we're just pointing out that the conclusion is not forced on us and can indeed be resisted (Korman 2016). Likewise, showing that for something to have a certain structure requires certain other things to be the case does not by itself prove that there are no structures. ${ }^{25}$

Finally, we adopted a structuralist approach to mathematics and presented the mapping account as the invocation of a proposed morphism between the structure of the target system and a mathematical structure, and our account of structure generating descriptions provided a story for how the former is generated. What becomes of our response to the general application problem if one changes the philosophy of mathematics and doesn't subscribe to structuralism? Are our structure generating descriptions useful from the perspective of other accounts of mathematics? We think so. But because of space limitations we can only gesture at some important cases.

For a Platonist, mathematics is the study of mathematical objects: the natural numbers are not a structure or pattern, but rather objects that exist independently in some isolated Platonic realm. But Platonic objects presumably still have a structure (even though they are not identified with structures), and hence the mapping account can get a grip on these objects in the same way as it does for the mathematical structuralist by prosing a mapping between the structure of Platonic objects and the structure of a target system as identified by DS.

For the formalist things look different. There is no subject matter of mathematics per se: mathematics is the study of systems of syntactic objects and rules for manipulating them. Mathematical application from this perspective can be thought of as providing a physical interpretation of the terms in the systems, and

\footnotetext{
${ }^{25}$ It's worth noting here that nothing we say in this paper has any bearing on other arguments for or against nominalism, for example appeals to the scientific practice of representing non-actual states of systems via phase spaces (Lyon and Colyvan 2008), or whether, once a representation theorem has been proven, everything that might be said about the mathematical structure can be rephrased in terms of the empirical structure (see Balaguer's (1998, p. 112) and Pincock's (2007, Sec. III) for further discussion).
} 
investigating which conclusions are generated by applying the rules to the result. If so, our $\boldsymbol{D}_{\boldsymbol{s}}$ can be thought of as specifying which physical objects and properties feature in the interpretations (this is reminiscent of Hilbert's remark that in a proper axiomatisation of geometry "one must always be able to say, instead of 'points, straight lines, and planes', 'tables, chairs, and beer mugs'”). ${ }^{26}$ Similar considerations apply to what Shapiro (1983, pp. 529-531) calls "postulate logicism", i.e. the study of the logical consequences of uninterpreted sets of axioms. Again, $\boldsymbol{D}_{\boldsymbol{s}}$ provides a way of interpreting the axioms physically, and the logical consequences of these axioms are the claims generated by the application of mathematics.

For the intuitionist, mathematical statements are about mathematical objects, but these are mental constructs. How to think about mathematical application in this framework is not straightforward, but one possibility it is to adopt a Kantian perspective whereby the human mind plays an active role in perceiving and understanding the world (Shapiro 1983, p. 533). Our $\boldsymbol{D}_{\boldsymbol{s}}$ then don't describe an objective world, but describe a world as perceived and understood by a human mind. An intuitionist about mathematics could then try to account for the application of mathematics by establishing a relationship between mathematical mental constructs and perceptual mental constructs, constructs as provided by $D_{s}$.

Each of these positions deserves further research. Regardless of the philosophy of mathematics adopted, one would hope that applications of mathematics would distinguish between applying mathematics to the system under one description and under another. By explicating this we hope to have made inroads in understanding how mathematics applies to the physical world.

\section{Acknowledgments}

We would like to thank the participants in JN's 'Models and Representation' Spring 2016 research seminar at the University of Notre Dame, Otávio Bueno, Mark Colyvan, Susana Lucero, and two anonymous referees for helpful discussions and/or comments on earlier drafts. Thanks also to the audiences in Nikosia and Rostock for helpful feedback. Special thanks goes to Demetris Portides for inviting us to be part of this project.

\section{References}

Ainsworth, P. (2009). Newman's objection. British Journal for the Philosophy of Science, 60(1), 135-171.

Baker, A. (2005). Are there genuine mathematical explanations of physical phenomena? Mind, 114(454), 223-238.

Baker, A. (2009). Mathematical explanation in science. British Journal for the Philosophy of Science, 60(3), 611-633.

\footnotetext{
${ }^{26}$ Blumenthal attributes this quote to Hilbert in his biography included in (Hilbert 1935).
} 
Balaguer, M. (1998). Platonism and anti-Platonism in mathematics. Oxford and New York: Oxford University Press.

Bartels, A. (2006). Defending the structural concept of represenation. Theoria, 21(1), 7-19.

Brading, K., \& Landry, E. (2006). Scientific structuralism: presentation and representation. Philosophy of Science, 73(5), 571-581.

Brown, J. R. (1999). Philosophy of mathematics: a contemporary introduction to the world of proofs and pictures (2nd ed.). New York: Routledge

Bueno, O., \& Colyvan, M. (2011). An inferential conception of the application of mathematics. Nous, 45(2), 345 - 374.

Bueno, O., \& French, S. (2011). How theories represent. British Journal for the Philosophy of Science, 62(4), 857-894.

Cartwright, N. (1999). The dappled world. A study of the boundaries of science. Cambridge: Cambridge University Press.

Colyvan, M. (2001). The indispensability of mathematics. Oxford: Oxford University Press.

Da Costa, N. C. A., \& French, S. (2003). Science and partial truth: a unitary approach to models and scientific Reasoning. Oxford: Oxford University Press.

Diez, J. (1997a). A hundred years of numbers. An historical introduction to measurement theory 1887-1990 Part I. Studies in History and Philosophy of Science, 28(1), 167-185.

Diez, J. (1997b). A hundred years of numbers. An historical introduction to measurement theory 1887-1990 Part II. Studies in History and Philosophy of Science, 28(2), 237-265.

Enderton, H. B. (1972/2001). A mathematical introduction to logic (2nd ed.). San Diego and New York: Harcourt.

Field, H. (1980). Science without numbers: a defense of nominalism. Princeton University Press: Cambridge,MA.

French, S., \& Ladyman, J. (1999). Reinflating the semantic approach. International Studies in the Philosophy of Science, 13, 103-121.

Frigg, R. (2006). Scientific representation and the semantic view of theories. Theoria, 55(1), 49-65.

Frigg, R., \& Nguyen, J. (2017). Models and representation. In L. Magnani, \& T. Bertolotti (Eds.), Springer handbook of model-based science. Switzerland: Springer.

Frigg, R., \& Nguyen, J. (forthcoming). The turn of the valve: representing with material models. European Journal for Philosophy of Science.

Frigg, R., \& Votsis, I. (2011). Everything you always wanted to know about structural realism but were afraid to ask. European Journal for Philosophy of Science, 1(2), 227-276.

Galileo, G. (1623/1957). The assyer. In S. Drake (Ed.), Discoveries and opinions of Galileo. New York: Doubleday \& Co.

Hartmann, S. (1999). Models and stories in hadron physics. In M. Morgan, \& M. Morrison (Eds.), Models as mediators. Perspectives on natural and social science (pp. 326-346). Cambridge: Cambridge University Press.

Hilbert, D. (1935). Gesammelte abhandlungen. Berlin: Springer.

Hodges, W. (1997). A shorter model theory. Cambridge: Cambridge University Press. 
Ketland, J. (2004). Empirical adequacy and ramsification. The British Journal for the Philosophy of Science, 55(2), 287-300.

Korman, D. Z. (2016). Ordinary objects. In E. N. Zalta (Ed.), The Stanford Encyclopedia of Philosophy

Krantz, D. H., Luce, R. D., Suppes, P., \& Tversky, A. (1971). Foundations of measurement (Vol 1). New York and London: Academic Press.

Lyon, A., \& Colyvan, M. (2008). The Explanatory power of phase spaces. Philosophia Mathematica, 16(2), 227-243.

Maddy, P. (1995). Naturalism and ontology. Philosophia Mathematica, 3(3), 248270.

Malament, D. (1982). Review of "Science without numbers: a defense of nominalism" by Hartry Field. Journal of Philosophy, 79(9).

Morgan, M. (2001). Models, stories and the economic world. Journal of Economic Methodology, 8(3), 361-384.

Muller, F. A. (2011). Reflections on the revolution at stanford. Synthese, 183(1), 87-114.

Mundy, B. (1986). On the general theory of meaningful representation. Synthese, 67(3), 391-437.

Newman, M. H. A. (1928). Mr. Russell's "causal theory of perception". Mind, 37, 137-148.

Nguyen, J. (2016). On the pragmatic equivalence between representing data and phenomena Philosophy of Science, 83(2), 171-191.

Oddie, G. (2016). Truthlikeness. In E. N. Zalta (Ed.), The Stanford Encyclopedia of Philosophy

Pero, F., \& Suárez, M. (2016). Varieties of misrepresentation and homomorphism. European Journal for Philosophy of Science, 6(1), 71-90.

Pincock, C. (2004). A new perspective on the problem of applying mathematics. Philosophia Mathematica, 12(3), 135-161.

Pincock, C. (2007). A role for aathematics in the physical sciences. Nous, 41(2), 253-275.

Pincock, C. (2012). Mathematics and scientific representation. Oxford: Oxford University Press.

Portides, D. (2017). Models and theories. In L. Magnani, \& T. Bertolotti (Eds.), Springer handbook of model-based science (pp. 25-48). Berlin and New York: Springer.

Resnik, M. D. (1997). Mathematics as a science of patterns. Oxford: Oxford University Press.

Shapiro, S. (1983). Mathematics and reality. Philosophy of Science, 50(4), 523548.

Shapiro, S. (1997). Philosophy of mathematics: structure and ontology. Oxford: Oxford University Press.

Steiner, M. (1998). The applicability of mathematics as a philosophical problem. Cambridge, MA and London: Harvard University Press.

Suppes, P. (1960). A comparison of the meaning and uses of models in mathematics and the empirical sciences. In P. Suppes (Ed.), Studies in the methodology and foundations of science: selected papers from 1951 to 1969 (pp. 10-23). Dordrecht Reidel 1969. 
Suppes, P. (1962/1969). Models of data. In P. Suppes (Ed.), Studies in the methodology and foundations of science: selected papers from 1951 to 1969 (pp. 24-35). Dordrecht: Dordrecht.

Tegmark, M. (2008). The mathematical universe. Foundations of Physics, 38(2), 101-150.

van Fraassen, B. C. (1980). The scientific image. Oxford: Oxford University Press.

van Fraassen, B. C. (2008). Scientific representation: paradoxes of perspective. Oxford: Oxford University Press.

Weisberg, M. (2013). Simulation and similarity: using models to understand the world. Oxford: Oxford University Press.

Wigner, E. (1960). The unreasonable effectiveness of mathematics in the natural sciences. Communications on Pure and Applied Mathematics, 13(1), 1-14. 